\title{
Immunohistochemical analysis of periodontal reattachment on denuded root dentin after periodontal surgery
}

\author{
Hiroyuki Harahashi ${ }^{1}$, Tomoomi Odajima ${ }^{1}$, Tsuneyuki Yamamoto $^{2}$, and Masamitsu Kawanami ${ }^{1}$ \\ Departments of ${ }^{1}$ Periodontology and Endodontology and ${ }^{2}$ Developmental Biology of Hard Tissue, Division of Oral Health Science, \\ Hokkaido University Graduate School of Dental Medicine, Kita 13, Nishi 7, Kita-ku, Sapporo 060-8586, Japan
}

(Received 5 July 2010; and accepted 20 August 2010)

\begin{abstract}
This study evaluates the effects of three kinds of periodontal surgery using statistical analyses of histological and immunohistochemical indices. Dehiscence defects were made on roots of maxillary teeth in monkeys. Surgically exposed roots were untreated, etched with EDTA, or treated with Emdogain $^{\circledR}$ after EDTA etching. Paraffin sections were stained with hematoxylin and eosin or immunostained for bone sialoprotein (BSP) or osteopontin (OPN) and analysed using several indices. The relative length of regenerated cementum and of BSP/OPN-immunoreactive lines on dentin defect showed no differences among the three groups. The attached regenerated cementum in the untreated group was shorter than in the etching groups. Regenerated cementum area was larger in the etching-Emdogain group than in the etching group. Thickness of immunolabeling on detached cementum was larger than that on attached cementum in all of the groups. These findings suggest that etching reinforces the attachment of regenerated cementum, and that BSP and OPN are associated with the attachment, where they exercise strong adhesion within a certain level of thickness.
\end{abstract}

The goal of periodontal treatment is to make damaged periodontal tissues recover their original structure and function. For this purpose, cementum regeneration on exposed roots and periodontal ligament regeneration with functional arrangement of collagen fibers are necessary, together with the regeneration of supporting alveolar bone. To date, many investigators have applied various kinds of clinical treatments to accelerate periodontal tissue regeneration $(13,14,20,22)$. However, in these studies, the regenerated cementum frequently detached from the root surface $(13,14,20,22)$. Some reports suggest that the detachment occurs at a col-

Address correspondence to: Hiroyuki Harahashi, Department of Periodontology and Endodontology, Division of Oral Health Science, Hokkaido University Graduate School of Dental Medicine, Kita 13, Nishi 7, Kita-ku, Sapporo 060-8586, Japan

Tel: +81-011-706-4266, Fax: +81-011-706-4266

E-mail: harahashi@den.hokudai.ac.jp lagen fiber-poor layer between the regenerated cementum and the root surface $(4,16,17)$. Generally, the original cementum is firmly attached to the root surface. This suggests that the adhesion mode of regenerated cementum induced by periodontal treatment is different from that of original cementum formed under normal development $(10,16,17)$.

Human and rat cementum contains two types of non-collagenous acid glycoproteins, bone sialoprotein (BSP) and osteopontin (OPN) $(12,18,24)$, which accumulate especially at the cemento-dentinal junction (7, 25-27). Densely accumulated BSP and OPN at the cemento-dentinal junction have been suggested to be involved in the induction of cementogenesis and in the firm adhesion of cementum and dentin in rat and human teeth $(2,7,11,25-27)$. Therefore, these glycoproteins could be used for new indices to evaluate cementum regeneration. In addition to the glycoproteins, collagen fibers may be counted as adhesive factors. Some studies have suggested that citric acid or EDTA etching after root 
planing accelerates the first step of periodontal reattachment, as surgically exposed collagen fibers are expected to intermingle with newly generated fibrous tissue $(1,5,23)$. Further, recent clinical studies have reported that enamel matrix derivative (EMD) accelerates the regeneration of cementum, which is structurally similar to the original cementum $(3,15)$.

This study was designed to evaluate the effects of different kinds of root surface treatments on the healing of periodontal tissues, especially on the adhesion of regenerated cementum and dentin, by analyzing several new indices combined with BSP and OPN immunohistochemistry. For this purpose, three types of periodontal surgery-non-application, EDTA etching, and EMD application after EDTA etching-were applied to dehiscence defects on maxillary tooth roots of monkeys. Several indices were measured, calculated in paraffin sections, and statistically analyzed.

\section{MATERIALS AND METHODS}

This study was conducted in accordance with the Guidelines of the Experimental Animal Committee, Hokkaido University Graduate School of Dental Medicine (No. 08-0087).

Experimental animals and teeth. Two male monkeys (Macaca fuscata; body weight, 8.4 and $8.8 \mathrm{~kg}$ ) were used. A total of 36 portions were examined in maxillary tooth roots of the two animals: the right and left canines (a total of 4 roots), the right and left first and second premolars (a total of 16 roots), and the right and left first and second molars (a total of 16 roots). For 3 months before surgery, the periodontal tissues of the animals had been kept healthy as follows: under general anesthesia by intramuscular injection of Ketamine $(0.25 \mathrm{~mL} / \mathrm{kg})$ (Ketalar $50^{\circledR}$, Sankyo, Tokyo, Japan) and medetomijin $(0.25 \mathrm{~mL} /$ kg) (Domitor ${ }^{\circledR}$, Meiji Seika, Tokyo, Japan), the teeth were brushed and scaled, and washed with $0.2 \%$ chlorhexidine gluconate (Hibitane ${ }^{\circledR}$, Sumitomo Confectionery, Tokyo, Japan) once or twice a week.

Experimental methods. Dehiscence defects were made on the buccal surface of the roots under a combination of general anesthesia (described above) and local anesthesia with $2 \%$ lidocaine hydrochloride containing 1/80,000 epinephrine (Xylocaine ${ }^{\circledR}$; AstraZeneca, Osaka, Japan). After exposure of the alveolar bone by full thickness flap operation, a dehiscence defect $(0.5 \mathrm{~mm}$ in width and $4 \mathrm{~mm}$ in length) reaching root dentin through the alveolar bone, periodontal ligament, and cementum was made from the cemento-enamel junction (CEJ) toward the root apex with a $1 / 4$ round bar $(0.5 \mathrm{~mm}$ diameter $)$ (Fig. 1a).

The operated portions were divided into three groups. In the Fop group, the exposed roots were untreated. In the Etc group, the exposed roots were etched with 24\% EDTA for 15 s. In the EMD group, the exposed roots were treated with Emdogain ${ }^{\circledR}$ gel (Seikagaku Kogyo, Tokyo, Japan) after the EDTA etching. The flap was restored to the original position and sutured immediately after the operation.

Pre- and post-operative control. To prevent infection, ampicillin sodium (300 mg/kg) (Viccillin-S500 ${ }^{\circledR}$; Meiji Seika, Tokyo, Japan) was administered by intramuscular injection once a day. Pellet food (Nippon Kurea, Tokyo, Japan) had been given once a day for 3 months before surgery. For 1 week after surgery, soft food was given. After this 1-week interval, the stitches were removed, and mouth cleaning and pellet food supply started again.

Observation methods. At the end of the observation period (3 months), the animals were perfused with $10 \%$ buffered formalin under general anesthesia. The teeth were removed together with surrounding tissues, and immersed in the same fixative. The specimens were demineralized in 10\% EDTA for 90 days and embedded in paraffin. Serial, $6-\mu \mathrm{m}$-thick, transverse sections of the root were cut from the CEJ toward the root apex.

\section{(1) Hematoxylin and eosin (H-E) staining}

Before deparaffinization, all sections were examined and both cervical and apical ends of the defect were determined. Then, sections were selected so that similar positions could be observed in all specimens. Firstly, one section was selected at the middle of each defect. Next, one section was selected as a cervical middle section at the cervical quarter of each defect, and one section was selected as an apical middle section at the apical quarter. The three sections of each defect, the middle section, the cervical middle section, and the apical middle section, were stained with H-E to examine the healing and regeneration of the periodontal tissues (Fig. 1b).

(2) Immunohistochemistry

Three serial sections (a total of 18 sections) were selected on both the cervical and apical sides of the middle, cervical middle, and apical middle sections in each defect. Nine sections of the cervical side 

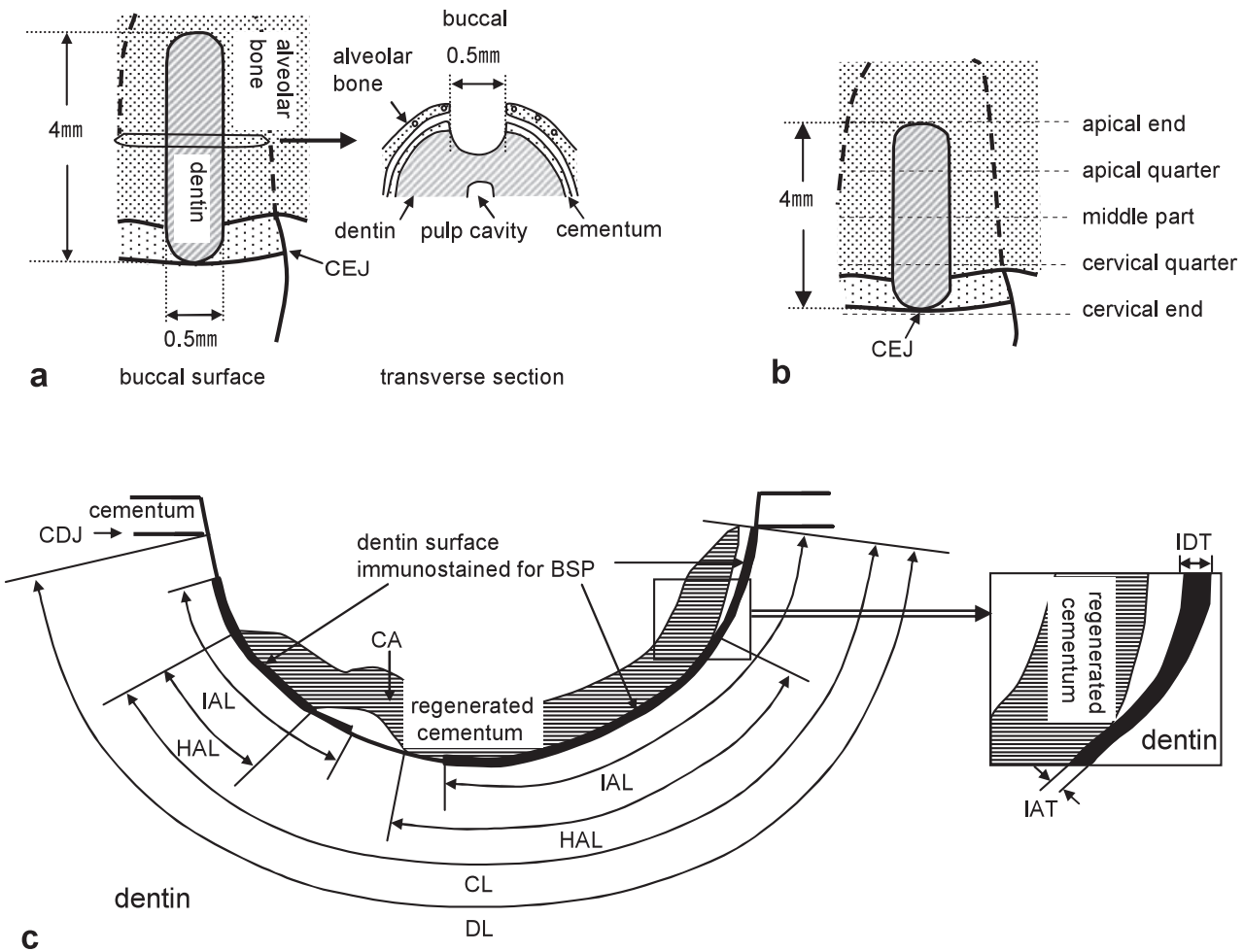

Fig. 1 Schematic diagrams showing the dehiscence defect (a), selected parts of sections (b), and measured indices (c). CDJ, cemento-dentinal junction; DL, defect length; CL, cementum length; HAL, histological attachment length; IAL, immunohistochemical attachment length; CA, cementum area; IAT, immunohistochemical attachment thickness; IDT, immunohistochemical detachment thickness

were stained for BSP, and the other nine sections of the apical side were stained for OPN (Fig. 1b). After deparaffinization, the sections were immersed in methanol containing $0.3 \% \mathrm{H}_{2} \mathrm{O}_{2}$ to block endogenous peroxidase activity, and then treated with $2.5 \%$ hyaluronidase (Sigma, Saint Louis, USA) at $37^{\circ} \mathrm{C}$ for $1 \mathrm{~h}$. The sections were then incubated with rabbit anti-human BSP polyclonal antibody (1:100) (Chemicon, Temecula, Canada) or rabbit anti-mouse OPN polyclonal antibody (LSL, Tokyo, Japan) for $1 \mathrm{~h}$, followed by biotinylated swine anti-rabbit IgG polyclonal antibody $(1: 100)$ (DAKO, Glostrup, Denmark) for $1 \mathrm{~h}$, and streptavidin-horseradish peroxidase complex (DAKO) for $30 \mathrm{~min}$. Finally, the immunoreaction was visualized by the 3,3'-diaminobenzidine method. For the negative controls, normal rabbit serum was substituted for the primary antibodies.

(3) Tissue measurement

Under a light microscope, images were taken at magnification of $100 \times(10 \times 10)$ using a personal computer. As the staining pattern of BSP and OPN was very similar in each defect, only BSP-stained sections were used for tissue measurement.
(4) Indices (Fig. 1c)

Measured indices were as follows: defect length (DL), the total length of the dentin surface defect; cementum length (CL), the length of the dentin surface defect with regenerated cementum; histological attachment length (HAL), the length of the dentin surface defect with attached (non-separated) regenerated cementum; immunohistochemical attachment length (IAL), the length of the dentin surface defect immunolabelled for BSP; cementum area (CA), the area of regenerated cementum $\left(\mu \mathrm{m}^{2}\right)$; immunohistochemical attachment thickness (IAT), the thickness of immunolabelling on attached regenerated cementum; and immunohistochemical detachment thickness (IDT), the thickness of immunolabelling on detached regenerated cementum.

Calculated indices were as follows: cementum regeneration rate, $\mathrm{CL} / \mathrm{DL} \times 100$; morphological attachment rate, total of HAL / DL $\times 100$; and immunohistochemical attachment rate, total of IAL / DL $\times 100$. Kruskal-Wallis test was used to test the significant differences among the three groups, and Mann-Whitney U-test was used to test those between two groups. 


\section{RESULTS}

\section{$H$-E staining}

Regenerated alveolar bone was continuous with the original bone in all groups. In most of the specimens, the regenerated bone covered the defect incompletely (Figs. 2a, 3, 4a, Table 1). Where the alveolar bone regenerated, periodontal ligament-like fibrous tissue formed between the regenerated bone and root. There was no displacement of root resorption or inflammatory root resorption in any of the groups. In all groups, the regenerated cementum covered more than half of the defect surface, but seldom covered the defect completely (Table 1). The original cementum adjacent to the defect was acellular cementum, while the regenerated cementum was cellular cementum, which was deposited thicker on the bottom of the defect than on the lateral wall (Figs. 2b, 2c, 3, 4b, 4c). A cementoblast layer was found on the regenerated cementum. In all groups, well-developed collagen fibers were perpendicularly embedded in the regenerated cementum on the lateral wall, while those fibers were less developed on the bottom (Fig. 2b, 2c). This tendency was most distinctly found in the EMD group (Fig. 4b, 4c). In all groups, the regenerated cementum-dentin junction as well as the original cemento-dentinal junction showed linear staining affinity for hematoxylin (Figs. 2c, 4c). The regenerated cementum, however, was frequently detached from the dentin or the original cementum, and the detachment was seen more frequently on the lateral wall than on the bottom (Figs. 2a, 3, 4a).

\section{Immunostaining}

Immunoreaction of BSP and OPN is shown in Figs. 5-7 and 8-10, respectively. The original cemento-dentinal junction showed intense immunoreactivities for BSP and OPN (Figs. 5b, 6b, 8b). In all groups, most of the regenerated cementum-dentin junction showed intense BSP and OPN immunoreactivities, which were similar to or more intense than that of the original cemento-dentinal junction (Figs. 5c, 6b, 7b, 8c, 9b, 10b), but occasionally showed no reactivity (Fig. 9b). Similar immunoreactivities were also found on the margin of detached regenerated cementum (Figs. 5a, 7a, 8a, 10a). Even where no regenerated cementum was found, the connective tissue frequently showed intense BSP and OPN immunoreactivities on the dentin surface (not shown). Control sections showed no specific immunoreactivity at all.
Statistical analysis

Among the three groups there was no significant difference in cementum regeneration rate (Table 2). Cementum area (CA) was significantly larger in the EMD group $(P<0.01)$ and the untreated (Fop) group $(P<0.05)$ than in the EDTA-etched $($ Etc) group $($ Table 3). Morphological attachment rate was significantly higher in the Etc group $(P<0.01)$ and the EMD group $(P<0.01)$ than in the Fop group, and was not different between the Etc and EMD groups (Table 4). The immunohistochemical attachment rate was not statistically different among the three groups (Table 5). The immunohistochemical detachment thickness (IDT) was significantly higher than the immunohistochemical attachment thickness (IAT) in all groups $(P<0.01)$ (Table 6).

\section{DISCUSSION}

The purpose of this study is to evaluate the effects of three different surgical techniques on the regeneration of periodontal tissues, especially on the attachment of regenerated cementum. For this purpose, several new indices that used BSP and OPN immunohistochemistry as well as conventional indices were measured, calculated, and statistically analyzed. The present study used monkeys because their oral morphology is very similar to that of human beings. Dehiscence defect was selected as the defect model, as the operation and standardization of this defect is relatively easy and a notch-shaped defect is favorable for regeneration of cementum $(1,14,16,20)$. Indeed, the cementum regeneration rate was more than $70 \%$ in all groups in this study (Table 2).

The cementum regeneration rate has been generally used as an index for cementum regeneration in previous studies $(13,15,20,22,23)$. However, as the detached cementum is also counted, this index does not strictly reflect the index of the regeneration of cementum attached strongly to the dentin. Therefore, this study was designed to calculate the morphological attachment rate as a more exact index of cementum regeneration. In addition, it is the first time to use BSP and OPN as indices, which glycoproteins have been suggested as adhesive substances, for evaluation of the attachment of regenerated cementum. As discussed in detail later, this study suggests that BSP and OPN are closely associated with the attachment between regenerated cementum and denuded dentin.

There was no significant difference in the cementum regeneration rate among the three groups. This suggests that either etching or EMD application has 

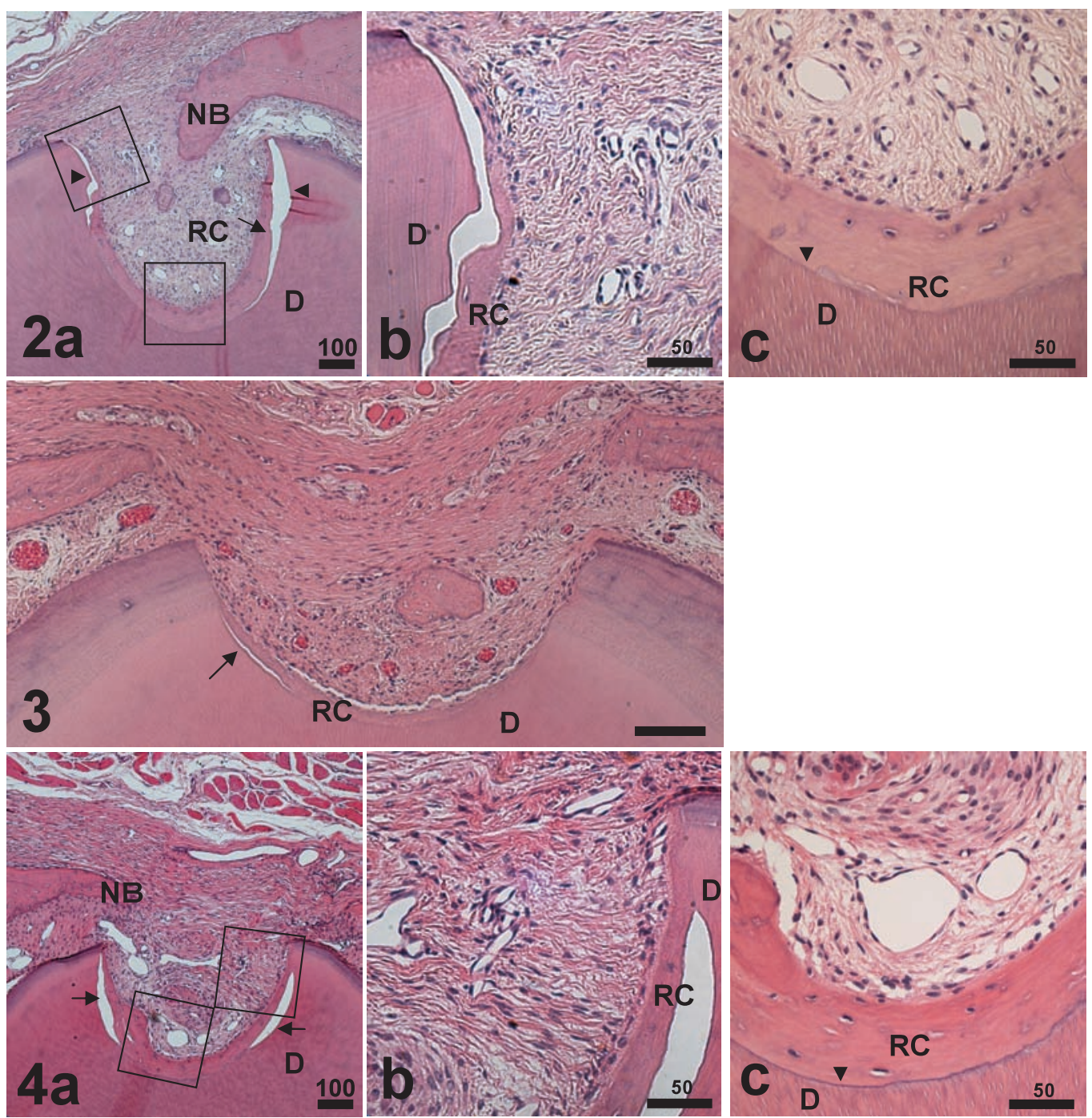

Fig. 2 The middle part of the defects in H-E stained sections in the untreated (Fop) group. a Overall view of the defect. Regenerated bone does not cover the defect completely. Regenerated cementum is detached on the lateral wall of the defect (arrowheads). b Highly magnified lateral wall of the defect (enclosed in a). Regenerated cementum is a cellular type. c Highly magnified bottom of the defect (enclosed in a). Collagen fibers are less regularly arranged than on the lateral wall. Regenerated cementum is a cellular type and thicker than on the lateral wall. Regenerated cementum-dentin junction (arrowhead) is stained with hematoxylin. $\mathrm{D}$, dentin; NB, regenerated new bone; RC, regenerated cementum.

Fig. 3 Overall view of the middle part of the defects in H-E stained sections in the EDTA-etched (Etc) group. Arrow indicates the detachment of regenerated cementum $(\mathrm{RC})$ on the lateral wall. Regenerated cementum is thicker on the bottom than on the lateral wall.

Fig. 4 The middle part of the defects in H-E stained sections in the EMD group. a Overall view of the defect. Regenerated cementum is detached on the lateral wall of the defect (arrows). b Highly magnified lateral wall of the defect (enclosed in a). Regenerated cementum is a cellular type. Well-developed collagen fibers are arranged perpendicularly to the regenerated cementum. c Highly magnified bottom of the defect (enclosed in a). Regenerated cementum is a cellular type and thicker than on the lateral wall. Collagen fibers are immature. Regenerated cementum-dentin junction (arrowhead) is stained with hematoxylin.

Table 1 Rate of complete regeneration of bone and cementum

\begin{tabular}{lccc}
\hline & Fop & Etc & EMD \\
& $(\mathrm{N}=10)$ & $(\mathrm{N}=11)$ & $(\mathrm{N}=10)$ \\
\hline New bone & $1 / 10$ & $1 / 11$ & $1 / 10$ \\
Regenerated cementum & $2 / 10$ & $0 / 11$ & $1 / 10$ \\
\hline
\end{tabular}



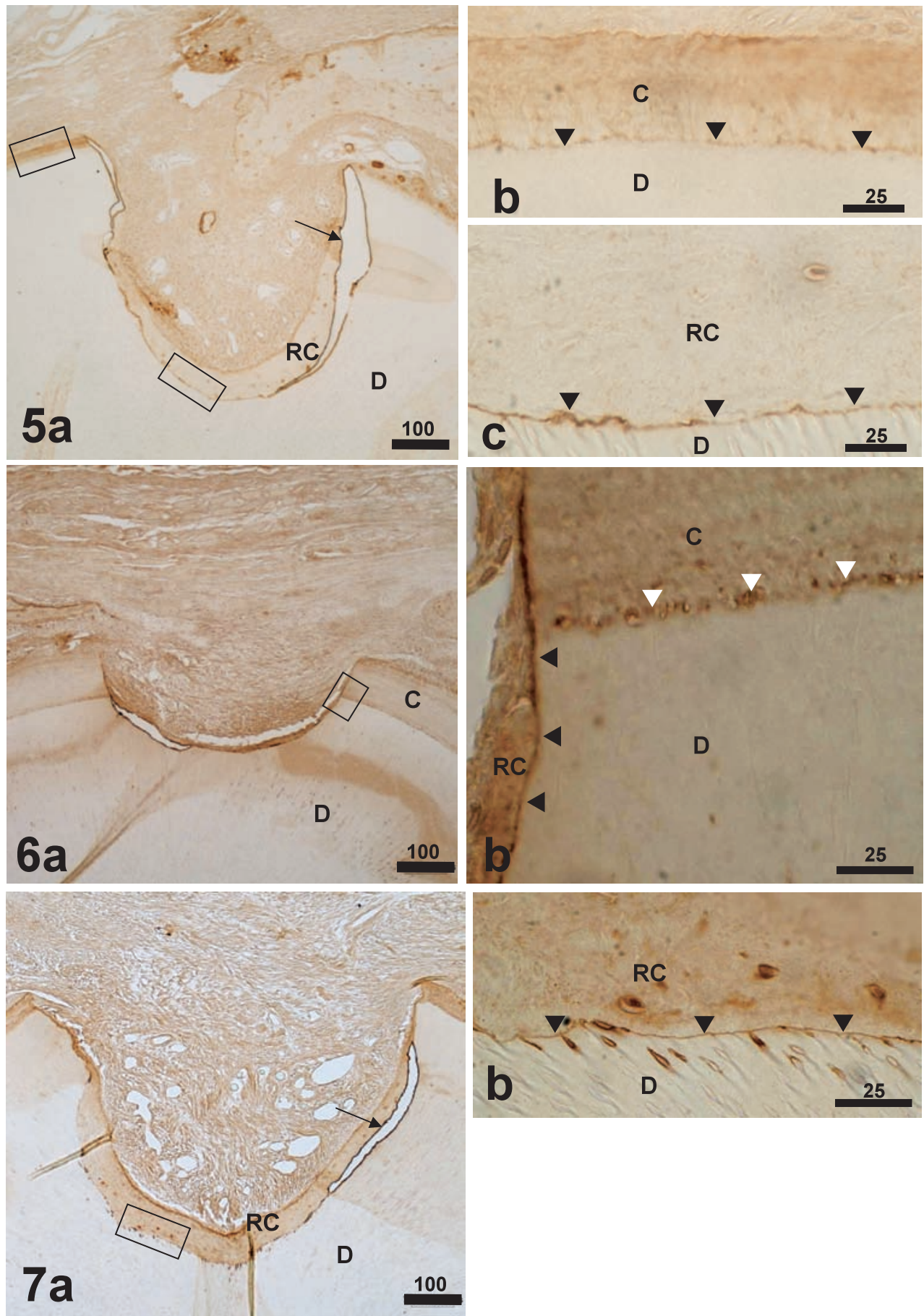

Fig. 5 The middle part of the defects in BSP-immunostained sections in the Fop group. a Overall view of the defect. Arrow indicates the immunoreactive margin of detached regenerated cementum. b Highly magnified unoperated portion closest to the orifice of the defect (enclosed in a). The original cemento-dentinal junction (arrowheads) is immunoreactive for BSP. c Highly magnified bottom of the defect (enclosed in a). The regenerated cementum-dentin junction (arrowheads) is heavily immunolabeled. C, cementum; D, dentin; RC, regenerated cementum.

Fig. 6 The middle part of the defects in BSP-immunostained sections in the Etc group. a Overall view of the defect. $\mathbf{b}$ Highly magnified lateral wall of the defect (enclosed in a). The original cemento-dentinal junction (white arrowheads) and regenerated cementum-dentin junction (black arrowheads) show an intense immunoreactivity.

Fig. 7 The middle part of the defects in BSP-immunostained sections in the EMD group. a Overall view of the defect. Arrow indicates the immunoreactive margin of detached regenerated cementum. b Highly magnified bottom of the defect (enclosed in a). The regenerated cementum-dentin junction (arrowheads) is immunoreactive. 

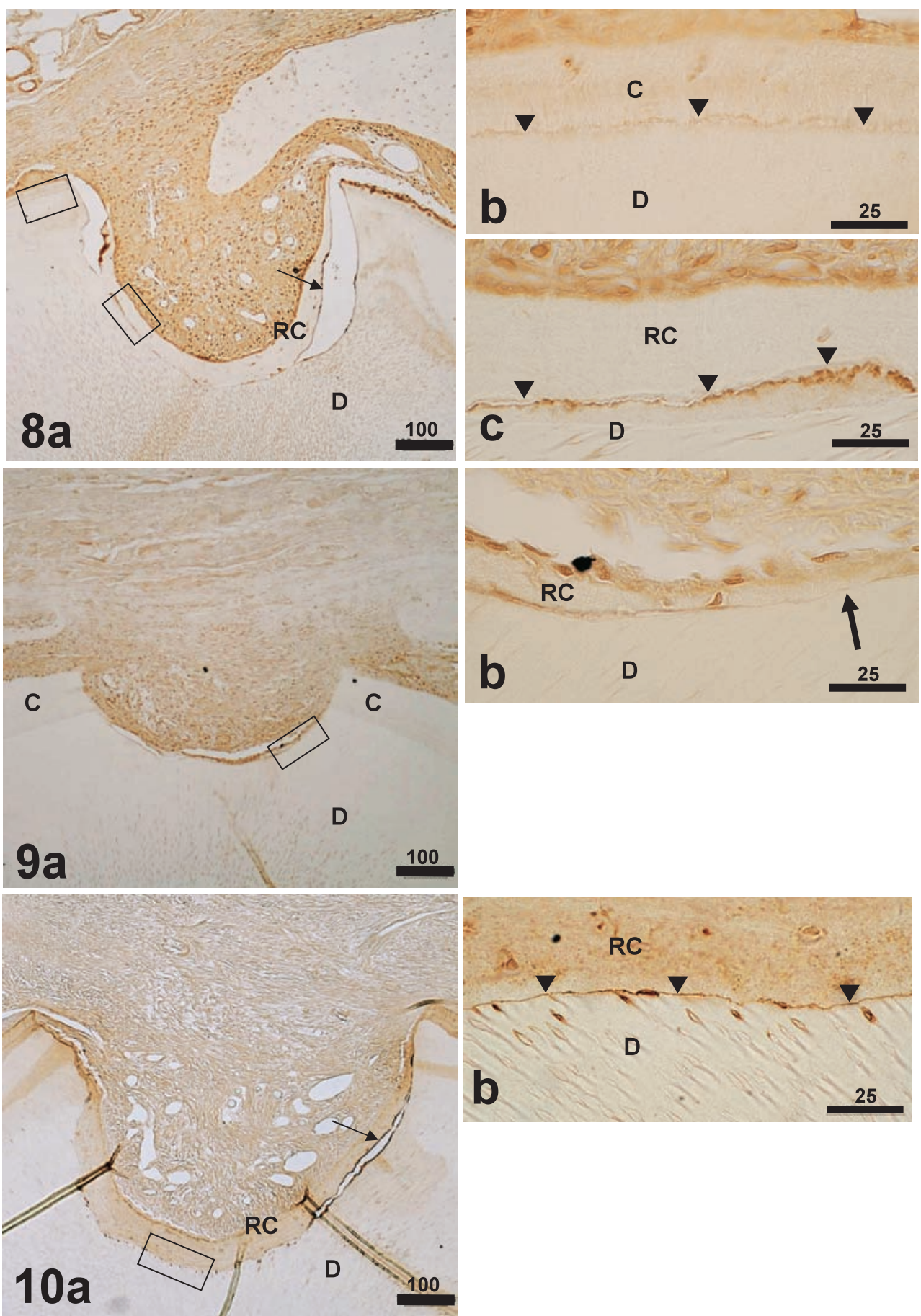

Fig. 8 The middle part of the defects in OPN-immunostained sections in the Fop group. a Overall view of the defect. Arrow indicates the immunoreactive margin of detached regenerated cementum. $\mathbf{b}$ Highly magnified unoperated portion closest to the orifice of the defect (enclosed in a). The original cemento-dentinal junction (arrowheads) is immunoreactive for OPN. c Highly magnified bottom of the defect (enclosed in a). The regenerated cementum-dentin junction (arrowheads) is immunolabeled. C, cementum; D, dentin; RC, regenerated cementum.

Fig. 9 The middle part of the defects in OPN-immunostained sections in the Etc group. a Overall view of the defect. $\mathbf{b}$ Highly magnified bottom of the defect (enclosed in a). Here regenerated cementum-dentin junction shows no immunoreactivity in some places (arrow).

Fig. 10 The middle part of the defects in OPN-immunostained sections in the EMD group. a Overall view of the defect. Arrow indicates the immunoreactive margin of detached regenerated cementum. b Highly magnified bottom of the defect (enclosed in a). The regenerated cementum-dentin junction (arrowheads) is intensely immunoreactive. 
Table 2 A statistical analysis of cementum regeneration rate

\begin{tabular}{|c|c|c|c|}
\hline & $\begin{array}{c}\text { Fop } \\
(\mathrm{N}=10)\end{array}$ & $\begin{array}{c}\text { Etc } \\
(\mathrm{N}=11)\end{array}$ & $\begin{array}{c}\text { EMD } \\
(\mathrm{N}=10)\end{array}$ \\
\hline \multirow{3}{*}{$\begin{array}{l}\text { Cementum } \\
\text { regeneration } \\
\text { rate (\%) }\end{array}$} & $74.46 \pm 19.38$ & $74.09 \pm 18.94$ & $77.96 \pm 12.47$ \\
\hline & & & \\
\hline & & N.S & \\
\hline
\end{tabular}

Kruskal-Wallis test: Not significant

Mann-Whitney's U-test: N.S (Not Significant)

Table 3 A statistical analysis of cementum area

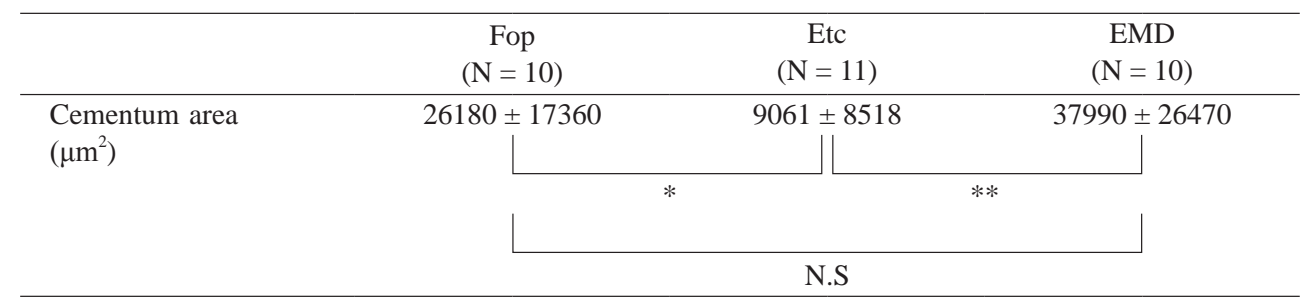

Kruskal-Wallis test: significantly different

Mann-Whitney's U-test: ** $(P<0.01) *(P<0.05)$

N.S (Not Significant)

Table 4 A statistical analysis of morphological attachment rate

\begin{tabular}{|c|c|c|c|}
\hline & $\begin{array}{c}\text { Fop } \\
(\mathrm{N}=10)\end{array}$ & $\begin{array}{c}\text { Etc } \\
(\mathrm{N}=11)\end{array}$ & $\begin{array}{c}\text { EMD } \\
(\mathrm{N}=10)\end{array}$ \\
\hline $\begin{array}{l}\text { Morphological } \\
\text { attachment rate }\end{array}$ & $14.70 \pm 9.67$ & $39.74 \pm 17.62$ & $41.37 \pm 16.14$ \\
\hline (\%) & & & \\
\hline
\end{tabular}

Kruskal-Wallis test: significantly different

Mann-Whitney's U-test: ${ }^{* *}(P<0.01)$ N.S (Not Significant)

Table 5 A statistical analysis of immunohistochemical attachment rate

\begin{tabular}{|c|c|c|c|}
\hline & $\begin{array}{c}\text { Fop } \\
(\mathrm{N}=10)\end{array}$ & $\begin{array}{c}\text { Etc } \\
(\mathrm{N}=11)\end{array}$ & $\begin{array}{c}\text { EMD } \\
(\mathrm{N}=10)\end{array}$ \\
\hline $\begin{array}{l}\text { Immunohistochemical } \\
\text { attachment }\end{array}$ & $93.00 \pm 7.060$ & $90.53 \pm 8 . .073$ & $93.56 \pm 6.981$ \\
\hline rate $(\%)$ & & & \\
\hline
\end{tabular}

Kruskal-Wallis test: significantly different

Mann-Whitney’s U-test: N.S (Not Significant)

no effect on the regeneration length of cementum. However, the regenerated cementum area was the smallest in the Etc group. It is likely that due to the narrow width of the defect, etching damaged sur- rounding tissues such as bone, periodontal ligament, and mucous flaps. Although similar etching was applied to the EMD group, the regenerated cementum area was larger than in the Etc group. A reason for 
Table 6 A statistical analysis of immunohistochemical attachment thickness and immunohistochemical detachment thickness

\begin{tabular}{lcc}
\hline & $\begin{array}{c}\text { Immunohistochemical attachment } \\
\text { thickness }(\mu \mathrm{m})\end{array}$ & $\begin{array}{c}\text { Immunohistochemical detachment } \\
\text { thickness }(\mu \mathrm{m})\end{array}$ \\
\hline $\begin{array}{l}\text { Fop } \\
(\mathrm{N}=10)\end{array}$ & $2.031 \pm 0.772$ \\
$\begin{array}{l}\text { Etc } \\
(\mathrm{N}=11)\end{array}$ & $1.786 \pm 0.442$ \\
$\begin{array}{l}\text { EMD } \\
(\mathrm{N}=10)\end{array}$ & $1.441 \pm 0.302$ \\
\hline
\end{tabular}

Mann-Whitney's U-test: ** $(P<0.01)$

Among the three groups there is no significant difference in the IAT or the IDT

this could be that the EMD application masked the harmful action of etching. In this study, regenerated cementum was all cellular cementum. This is consistent with previous reports that the cellular cementum is predominantly regenerated in experimental defects in human teeth (9), and that the EMD application induces cellular cementum formation in human (19) and monkey teeth (22). In some reports, however, EMD application in dog (3) and monkey teeth (15) induced acellular cementum formation. The findings obtained in this study were unable to provide us with a reason for this inconsistency.

The morphological attachment rate in the Etc and EMD groups was significantly higher than that in the Fop group: the regenerated cementum was detached more extensively in the Fop group than in the other two groups. The tissue sections examined in the present study were cut, selected, and stained under the same conditions in all groups. Therefore, the adhesive strength is suggested to be weaker in the Fop group than in the other groups. As there was no significant difference between the EMD and Etc groups in the morphological attachment rate, weaker attachment in the Fop group may be attributed to non-etching condition. There have been two opinions for the etching effects. First, the etching removes a smear layer and exposes the dentinal matrix fibers, resulting in that the fibers of regenerated cementum can be easily interwoven with those of dentin, to reinforce the cemento-dentinal attachment $(6,8)$. Second, some electron-dense substances, which inhibit the cemento-dentinal adhesion, remain on the dentin defect, but are removed by etching (6, 17, 21). At present it is unknown which opinion is correct.

In this study, the regenerated cementum was de- tached more frequently on the lateral wall of the defect than on the bottom in all groups. Thick substantial collagen fibers were organized on the lateral wall of the defect, while the collagen fibers were thin and immature on the bottom. Regenerated cementum on the bottom was thicker than on the lateral wall. During tissue preparation, thickly developed collagen fibers may be contracted more intensely than the immature fibers, which may result in more frequent detachment on the lateral wall.

The interface of regenerated cementum and dentin showed intense immunoreactivities for BSP and OPN with only a few exceptions. This suggests that the two glycoproteins are closely associated with the attachment of the two tissues. There was no significant difference among the three groups in immunohistochemical attachment rate. Therefore, both etching and EMD application may be ineffective on the occurrence of BSP- and OPN-mediated attachment and on prospective regeneration of cementum. The thickness of immunolabeling was significantly thicker on attached cementum than on detached cementum. This suggests that BSP and OPN strengthen the attachment within a certain deposition level (thickness), and the attachment becomes weaker beyond this level.

This study was done only at a light microscopic level. Therefore, some problems remain unsolved, thus raising the questions of whether the fibril intermingling of generated cementum and dentin increases in number after etching, and whether EMD application changes the denuded dentin surface structure. To examine the histological features of the attachment in more detail, immuno-electron microscopic observation as well as regular electron microscopic observations will be necessary. 
In conclusion, this study suggests that: 1) Etching reinforces the attachment of regenerated cementum and dentin, but may be harmful to the surrounding periodontal tissues. 2) BSP and OPN are closely associated with the attachment. In addition, thin membrane-like deposition of BSP and OPN exercises stronger adhesion than thick deposition of them. 3) It is considered that the morphological attachment rate and immunohistochemical indices using BSP and OPN will be helpful to evaluate cementum regeneration in future studies.

\section{REFERENCES}

1. Albair WB, Cobb CM and Killoy WJ (1982) Connective tissue attachment to periodontally diseased roots after citric acid demineralization. J Periodontol 53, 515-526.

2. Arambawatta AK, Yamamoto T and Wakita M (2005) Immunohistochemical characterization of noncollagenous matrix molecules on the alveolar bone surface at the initial principal fiber attachment in rat molars. Ann Anat 187, 77-87.

3. Araújo MG and Lindhe J (1998) GTR treatment of degree III furcation defects following application of enamel matrix proteins. An experimental study in dogs. J Clin Periodontol 25, 524-530.

4. Bjorn H (1961) Experimental studies on reattachment. Dent Pract 10, 351-354.

5. Blomlöf J, Blomlöf L and Lindskog S (1997) Effect of different concentrations of EDTA on smear removal and collagen exposure in periodontitis-affected root surfaces. J Clin Periodontol 24, 534-537.

6. Bosshardt DD, Degen T and Lang NP (2005) Sequence of protein expression of bone sialoprotein and osteopontin at the developing interface between repair cementum and dentin in human deciduous teeth. Cell Tissue Res 320, 399-407.

7. Bosshardt DD and Nanci A (1998) Immunolocalization of epithelial and mesenchymal matrix constituents in association with inner enamel epithelial cells. J Histochem Cytochem 46, 135-142.

8. Bosshardt DD and Schroeder HE (1991) Initiation of acellular extrinsic fiber cementum on human teeth. A light- and electron-microscopic study. Cell Tissue Res 263, 311-324.

9. Bosshardt DD and Schroeder HE (1992) Initial formation of cellular intrinsic fiber cementum in developing human teeth. A light- and electron-microscopic study. Cell Tissue Res 267, 321-335.

10. Bosshardt DD and Selvig KA (1997) Dental cementum; the dynamic tissue covering of the root. Periodontol 2000 13, 41-75.

11. Fukushima C, Yamamoto T, Wakita $\mathrm{M}$ and Kawanami $\mathrm{M}$ (2006) A histological and immunohistochemical study of the structure and function of the cemento-dentinal junction. Hok- kaido J Dent Sci 27, 159-170.

12. Ganss B, Kim RH and Sodek J (1999) Bone sialoprotein. Crit Rev Oral Biol Med 10, 79-98.

13. Gottlow J, Nyman S, Karring T and Lindhe J (1984) New attachment formation as the result of controlled tissue regeneration. J Clin Periodontol 11, 494-503.

14. Hammarström L (1997) Enamel matrix, cementum development and regeneration. J Clin Periodontol 24, 658-668.

15. Hammarström L, Heijl L and Gestrelius S (1997) Periodontal regeneration in a buccal dehiscence model in monkeys after application of enamel matrix proteins. J Clin Periodontol 24, 669-677.

16. Heijl L (1997) Periodontal regeneration with enamel matrix derivative in one human experimental defect. A case report. $J$ Clin Periodontol 24, 693-696.

17. Listgarten MA (1972) Electron microscopic study of the junction between surgically denuded root surfaces and regenerated periodontal tissues. J Periodontal Res 7, 68-90.

18. Luder HU and Zappa U (1998) Nature and attachment of cementum formed under guided conditions in human teeth. An electron microscopic study. J Periodontol 69, 889-898.

19. McKee MD, Zalal S and Nanci A (1996) Extracellular matrix in tooth cementum and mantle dentin; localization of osteopontin and other noncollagenous proteins, plasma proteins, and glycoconjugates by electron microscopy. Anat Rec 245, 293-312.

20. Nyman S, Gottlow J, Karring T and Lindhe J (1982) The regenerative potential of the periodontal ligament. An experimental study in the monkey. J Clin Periodontol 9, 257-265.

21. Ririe CM, Crigger M and Selvig KA (1980) Healing of periodontal connective tissues following surgical wounding and application of citric acid in dogs. $J$ Periodontal Res 15, 314327.

22. Sculean A, Donos N, Brecx M, Reich E and Karring T (2000) Treatment of intrabony defects with guided tissue regeneration and enamel-matrix-proteins. An experimental study in monkeys. J Clin Periodontol 27, 466-472.

23. Selvig KA, Sigurdsson TJ and Wikesjö UME (1995) “Collagen adhesion” revisited. J Periodont Rest 15, 529-537.

24. Sodek J, Ganss B and McKee MD (2000) Osteopontin. Crit Rev Oral Biol Med 11, 279-303.

25. Yamamoto T, Domon T, Takahashi S, Arambawatta AK, Anjuman KA, Fukushima C and Wakita M (2005) Determination of two different types of cellular cementogenesis in rat molars: a histological and immunohistochemical study. Matrix Biol 24, 295-305.

26. Yamamoto T, Domon T, Takahashi S, IsLam NM and Suzuki R (2000) The fibrous structure of the cemento-dentinal junction in human molars shown by scanning electron microscopy combined with NaOH-maceration. J Periodontal Res 35, 59-64.

27. Yamamoto T, Domon T, Takahashi S, IsLam NM, Suzuki R and Wakita M (2000) The structure of the cemento-dentinal junction in rat molars. Ann Anat 182, 185-190. 